\title{
COVID-19 en América Latina y Caribe: Determinación de prioridades en investigación y llamado a la acción
}

\author{
COVID-19 in Latin America and Caribbean: A determination of research priorities and call to action
}

\author{
Nicole Feune de Colombi, ${ }^{1}$ Flavia Bueno, ${ }^{2}$ Jackeline Alger, ${ }^{3}$ Bonny Baker, ${ }^{4}$ Julio Canario, ${ }^{5}$ Laureano Mestra, ${ }^{6}$ Lyda Osorio, ${ }^{7}$ \\ Orielle Solar Hormazabal, ${ }^{8}$ Mario E. Soriano, ${ }^{9}$ Gustavo Matta, ${ }^{10}$ Trudie Lang, ${ }^{11}$ Ramón J. Soto. ${ }^{12}$ \\ ${ }^{1} \mathrm{MD}, \mathrm{MSc}$; Centre for Tropical Medicine and Global Health, Nuffield Department of Medicine, University of Oxford, Oxford, Reino Unido. \\ ${ }^{2} \mathrm{PhD}, \mathrm{MSc}$; Fundación Oswaldo Cruz, Rio de Janeiro, Brasil. \\ ${ }^{3} \mathrm{MD}, \mathrm{PhD}$; Unidad de Investigación Científica, Facultad de Ciencias Médicas (FCM), Universidad Nacional Autónoma de Honduras (UNAH); Departamento de \\ Laboratorio Clínico, Hospital Escuela; Instituto de Enfermedades Infecciosas y Parasitología Antonio Vidal (IAV); Tegucigalpa, Honduras. \\ ${ }^{4}$ BSc, MSc; Centre for Tropical Medicine and Global Health, Nuffield Department of Medicine, University of Oxford, Oxford, Reino Unido. \\ ${ }^{5} \mathrm{MSc}$; Centro Nacional de Investigación en Salud Materno-Infantil Dr. Hugo Mendoza, Santo Domingo, República Dominicana. \\ ${ }^{6} \mathrm{MD}, \mathrm{MSc} ;$ Hospital General de Medellín Luz Castro de Gutiérrez ESE, Medellín, Colombia. \\ ${ }^{7} \mathrm{MD}, \mathrm{PhD}$; Escuela de Salud Pública, Universidad del Valle, Cali, Colombia. \\ ${ }^{8} \mathrm{MD}, \mathrm{MPH}, \mathrm{MSc}$, Facultad Latino Americana de Ciencias Sociales FLACSO-CHILE, Santiago, Chile. \\ ${ }^{9} \mathrm{MD}, \mathrm{MSc}, \mathrm{MPH}$; Unidad de Atención Integral de Adolescentes y Jóvenes, Ministerio de Salud, San Salvador, El Salvador. \\ ${ }^{10} \mathrm{PhD}, \mathrm{MPH}$; Escuela de Salud Pública, Fundación Oswaldo Cruz Foundation, Rio de Janeiro, Brasil. \\ ${ }^{11} \mathrm{PhD}$; Centre for Tropical Medicine and Global Health, Nuffield Department of Medicine, University of Oxford, Oxford, Reino Unido. \\ ${ }^{12} \mathrm{MD}, \mathrm{MHS}$; Programa de Maestría en Epidemiología, FCM, UNAH; IAV; Tegucigalpa, Honduras.
}

RESUMEN. Antecedentes: La Red de Salud Global estableció, en enero 2020, una comunidad de práctica para abordar la investigación en COVID-19 en países de ingresos bajos/medios. Objetivo: Identificar prioridades en investigación sobre COVID-19 que requieren atención urgente en América Latina y el Caribe (LAC) y establecer una comunidad de práctica abierta local para apoyar su implementación. Métodos: Estudio exploratorio mixto. Se analizaron los resultados específicos para LAC de una encuesta en línea (enfoque cuantitativo) que evaluó si la agenda prioritaria de investigación para COVID-19 de la OMS sigue siendo pertinente solicitando a los participantes que clasificaran sus tres principales prioridades a corto y largo plazo. Asimismo, se organizó un taller virtual abierto (enfoque cualitativo) el cual fue grabado. Se realizó un análisis temático pragmático a partir de las presentaciones de los panelistas y de las preguntas y comentarios de la audiencia. Se generó un marco de codificación mediante enfoques inductivo y luego deductivo siguiendo la agenda OMS. Resultados: Se contó con 223 participantes de 22 países. Se identificó un consenso sobre los temas de investigación e innovación prioritarios para LAC, dentro y fuera del marco de la agenda OMS, siendo una gran prioridad la necesidad de estudios de ciencias sociales para ayudar a los científicos biomédicos. Discusión: Dado que los casos siguen aumentando en LAC, consideramos que nuestros hallazgos son útiles para orientar tanto a las redes de investigación en la planificación de estudios como a los financiadores en sus decisiones para la asignación de recursos para investigación e innovación. Palabras clave. América Latina, Brechas de la práctica profesional, Infección por coronavirus, Investigación, Prioridades en salud, Región del Caribe.

Recibido: 24-08-2020 Aceptado para publicación 14-12-2020

Dirección para correspondencia: Dra. Nicole Feune de Colombi

Correo electrónico: nicole.feunedecolombi@ndm.ox.ac.uk

Declaración de relaciones y actividades financieras y no financieras y conflictos de interés: ninguno.

DOI: https://doi.org/10.5377/rmh.v88i2.11488

\section{INTRODUCCIÓN}

La ocurrencia del SARS-CoV-2 y su declaración como una emergencia de importancia internacional por la Organización Mundial de la Salud (OMS) el 30 de enero de 2020, y posteriormente como pandemia el 11 de marzo, ${ }^{1}$ presionó por una coordinación global de respuestas incluyendo el establecimiento de una agenda de investigación e innovación. Así, la OMS convocó a más de 300 expertos y financiadores de diversos países y estableció una agenda prioritaria de investigación e innovación identificando 9 categorías, con sus correspondientes prioridades. $^{2}$

Para los países del llamado Sur Global, ${ }^{3}$ la pandemia pone de relieve importantes desafíos tales como las desigualdades estructurales que limitan la implementación de las medidas, así como las deficiencias de los sistemas de vigilancia, de los propios sistemas de salud y de las políticas de sostenibilidad, para responder de forma rápida y eficaz a la emergencia sanitaria. Según expresan Ventura DFL y colaboradores, ${ }^{4}$ "Es evidente que la respuesta a la emergencia sólo es posible en presencia de sistemas de salud bien estructurados, con capacidad de vigilancia e investigación, llevados a cabo por una masa crítica de profesionales bien capacitados y entrenados para actuar en estas tres áreas".

Es transcendental que la investigación se lleve a cabo en todo el mundo, no sólo para abordar las prioridades y determinar las intervenciones de tratamiento y prevención, sino también para garantizar la equidad en aquellos que se beneficien de los resultados de las investigaciones. ${ }^{5}$ Para contribuir a la respuesta a la pandemia, La Red de Salud Global (The Global Health Network), ${ }^{6}$ una plataforma que alberga diferentes comu- 
nidades de práctica ${ }^{a}$ en áreas específicas de investigación y disponibilidad de una amplia gama de recursos para guiar, capacitar y apoyar a los investigadores en el diseño, planificación y operación de sus estudios, ${ }^{7}$ estableció en enero de 2020 un Nodo/HUB para la implementación de la investigación de COVID-19 en países de ingresos bajos/medianos. El Nodo se creó con el objetivo de proporcionar un acceso equitativo a información, herramientas, recursos y oportunidades para permitir la investigación durante la pandemia de COVID-19.

Desde sus inicios, la Red y sus aliados estratégicos, se han enfocado en identificar barreras y facilitadores que permitan abordar y fortalecer la capacidad de investigación en países de bajos/medianos ingresos, ${ }^{8-10}$ incluyendo en situaciones emergentes como fueron las epidemias de Ébola y Zika. ${ }^{11}$ Con dicha experiencia y utilizando su plataforma, la Red organiza periódicamente actividades interactivas virtuales regionales para facilitar el debate en torno a las prioridades de investigación, las preguntas sin respuesta relacionadas con la evolución de la pandemia y los esfuerzos de investigación en entornos de bajos recursos. En ese sentido, la plataforma se inserta en el marco de lo que se considera una innovación social, es decir, una "solución innovadora (proceso, producto, práctica, mecanismo de mercado) desarrollada por un actor, en respuesta a una prioridad de salud enmarcada en un contexto geográfico e implementada por diferentes organizaciones intersectoriales". ${ }^{12}$

El presente estudio exploratorio con abordaje mixto se realizó con el objetivo de identificar prioridades en investigación sobre COVID-19 que requieren atención urgente en América Latina y el Caribe (LAC) y establecer una comunidad de práctica abierta para apoyar su implementación. En este artículo se describen los resultados identificados presentándose como un llamado a la acción para fomentar una mayor colaboración en su abordaje, no solamente durante esta pandemia, sino aumentando la preparación de los países ante situaciones similares futuras.

\section{PARTICIPANTES Y MÉTODOS}

En marzo 2020, la Academia Africana de Ciencias (AAS) realizó un ejercicio para evaluar hasta qué punto las prioridades dentro de cada una de las 9 categorías de la OMS eran aplicables a África. ${ }^{13}$ En este estudio, se hallaron 32 prioridades nuevas. Basados en este ejercicio de comparación de prioridades con las de la OMS, es que desarrollamos la metodología para este estudio. Realizamos un estudio exploratorio utilizando métodos mixtos. Se obtuvo información a través de un taller virtual abierto (análisis cualitativo) y a través de una encuesta en línea (análisis cuantitativo), entre abril y julio de 2020.

El 24 de abril del 2020, el Taller virtual "COVID-19 para países de habla hispana" se organizó conjuntamente con la Fundación Oswaldo Cruz (Fiocruz) y la Red de Capacidad de Investigación (REDe). Se identificaron oradores y moderadores de seis países de LAC (coautores) y el evento se difundió

${ }^{a}$ Comunidades de práctica son centros o Nodos (Hubs) en los que investigadores de todo el mundo se reúnen y comparten sus conocimientos en diferentes enfermedades y áreas/disciplinas de investigación. ampliamente en canales de comunicación de TGHN, Fiocruz, REDe, así como a través de las redes locales en cada país participante. La página de registro incluía el programa del taller y una breve reseña de las cuestiones que se debatirían, como se detalla a continuación: i) ¿Cómo actúa su país ante el brote de COVID-19 (vigilancia, diagnóstico, nuevas intervenciones, prevención, medidas gubernamentales)? ii) ¿Cuáles son las brechas de investigación que necesitan atención urgente? iii) ¿Utiliza o conoce alguna herramienta en español que podría ser útil para responder al escenario de la pandemia?

Con el fin de lograr un rápido consenso de los resultados, se utilizó una metodología de análisis de contenido temático inductivo desarrollada por TGHN para el análisis y el informe de las conclusiones de cada uno de sus talleres periódicos. ${ }^{14}$ Los datos incluidos en el análisis comprenden resúmenes generales y de situación presentados por los panelistas y los comentarios de los participantes (función "Pregunta y Respuesta" y "Chat"). Tras la inmersión de los datos, se generó un marco de codificación por medio de un proceso iterativo, refinando el número hasta lograr la saturación. Se identificaron unos verbatim que se utilizaron como representativos temáticos. Luego, se codificaron los temas de manera deductiva en relación a las 9 categorías utilizadas en la agenda prioritaria de investigación de la $\mathrm{OMS},{ }^{2}$ evaluando si los temas identificados ya se encontraban dentro de dichas categorías existentes o si nuevas prioridades fueron identificadas.

Luego del taller, en conjunto con la Academia Africana de Ciencias (AAS) y UK Collaborative Development Research, se desarrolló una encuesta en línea en junio 2020 evaluando 73 prioridades - las 41 del documento original de la OMS y las 32 generadas de la encuesta de la AAS en marzo (las prioridades encontradas en la encuesta de la AAS se organizaron bajo las 9 categorías utilizadas en la hoja de ruta de la OMS). Se pidió a los participantes que numeraran de 1 al 3 , siendo 1 la mayor prioridad, sus tres principales prioridades a corto y largo plazo dentro de cada una de las 9 categorías.

El consentimiento para participar se hizo en forma de "consentimiento implícito"b para los individuos que eligieron participar a través del "optar por continuar" para completar la encuesta. La encuesta se diseminó en varios idiomas y logró un alcance mundial con 1528 respuestas de 137 países. ${ }^{15}$ Las respuestas se descargaron en formato Excel, de forma anónima, protegidos con contraseña y acceso restringido al equipo de estudio. Se separaron las respuestas de la encuesta correspondientes a participantes de LAC, llevando a cabo un análisis estadístico descriptivo en formato Excel, donde se ponderaron las prioridades para proporcionar una puntuación de clasificación para cada prioridad de investigación a corto y largo plazo.

\footnotetext{
b "En el caso de ciertos tipos de investigación de bajo riesgo, como la realización de un simple cuestionario en línea, la realización de, y la presentación del cuestionario implica que el consentimiento para el uso de los datos del cuestionario se le ha dado. Sin embargo, el cuestionario debe ir precedido de información escrita sobre la y sus objetivos (incluida la información sobre cómo se almacenarán y publicarán los datos y una marca de verificación que confirme que los participantes tienen 18 años o más y aceptan participar)." Guía de mejores prácticas del Comité de Ética de la Investigación de la Universidad Central (CUREC) para la "Investigación basada en la Internet". 06_Versión 6.5 Aprobada por CUREC, Universidad de Oxford. Noviembre de 2019.
} 
Reconociendo que esta encuesta se centraba inherentemente en evaluar las ya existentes prioridades de la OMS, nuestra consulta se amplió mediante los resultados del taller interactivo.

\section{RESULTADOS}

En total, combinando el taller y la encuesta, 22 países de LAC fueron representados en este estudio (Figura 1).

Respecto a la encuesta, 93 personas de 17 países de LAC, la completaron. Los participantes representaron una amplia demografía de la investigación clínica, que abarcó una gran variedad de funciones laborales, incluidos puestos de nivel subalterno (por ejemplo, digitadores) y superior (por ejemplo, director de proyectos, $20 \%$, e investigadores superiores), con aportaciones de múltiples disciplinas incluidas epidemiología $(60 \%)$, de laboratorio $(29 \%)$, de ciencias sociales (18\%) y de asesoramiento político $(15 \%)$. Los participantes trabajaban principalmente en hospitales $(35 \%)$ o academia (30\%). Sin embargo, en varios tipos de establecimientos se demostró que estaban representados los puntos de vista, las aptitudes y las preocupaciones de los investigadores de diferentes sectores e industrias. Finalmente, $29 \%$ eran expertos en relación al control de enfermedades infecciosas.

A través de esta encuesta, se identificaron las principales prioridades en materia de investigación sobre COVID-19 que

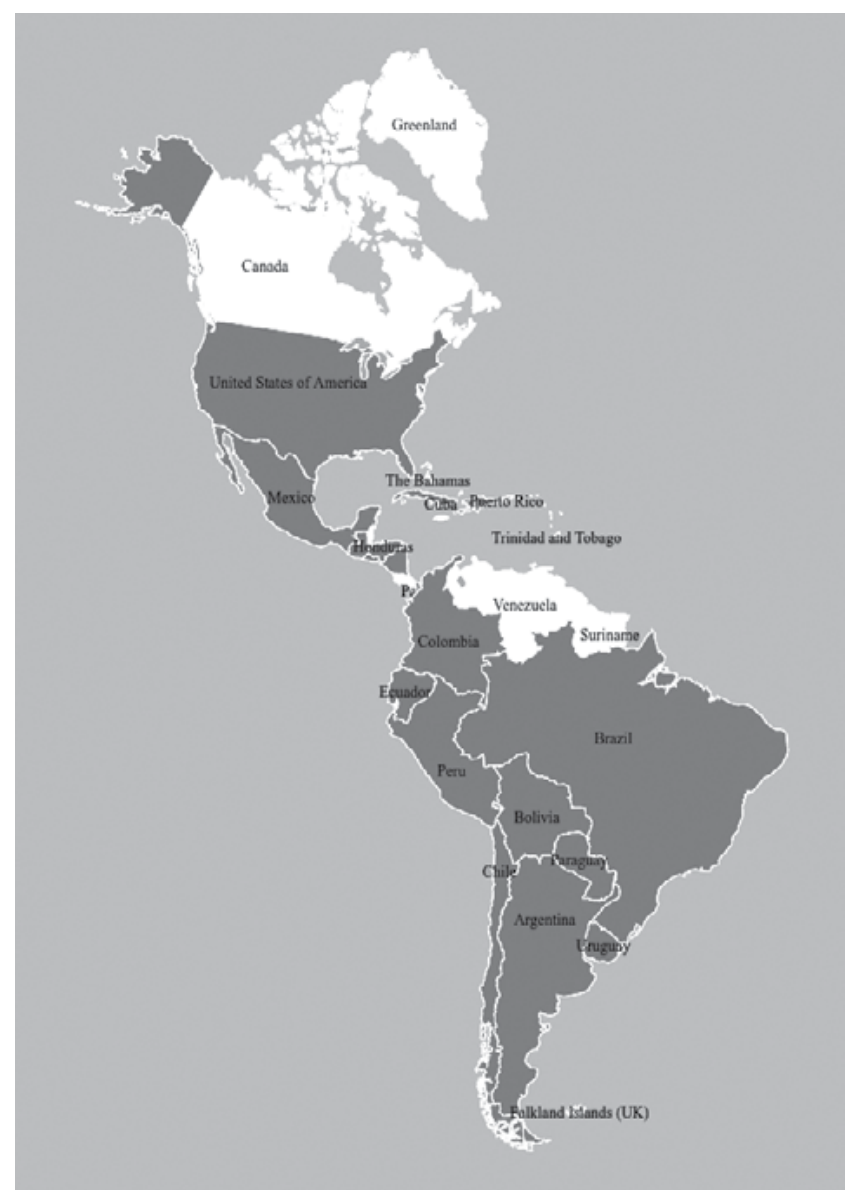

Figura 1. Ubicación de los participantes del taller virtual y de la encuesta en línea (países sombreados), La Red de Salud Global, abril - junio 2020. requieren una atención urgente en LAC. El Cuadro 1 muestra las tres principales prioridades a corto y largo plazo dentro de cada una de las 9 categorías de la hoja de ruta de la OMS.

Más de 250 personas se inscribieron para asistir al taller. Ya que la cantidad de registros excedía la capacidad de la plataforma digital utilizada, invitamos al máximo número de participantes que podían ser acomodados a la videoconferencia Zoom. En total, 130 participantes más panelistas de 19 países finalmente participaron en el taller. Partiendo de las preguntas guía utilizadas para promover el evento, tuvimos la oportunidad de revisar el panorama de COVID-19 en cada país participante y, además de compartir experiencias específicas. Observamos que muchas de las prioridades de investigación señaladas son comunes en muchos países. Si bien es cierto que cada país tiene sus sistemas de salud y sus poblaciones con contextos diversos, también hay diversas similitudes que nos aproximan y que podemos solucionar de forma conjunta y coordinada.

Del análisis cualitativo de las presentaciones y de la discusión durante el taller, surgieron las siguientes prioridades de investigación en la región en el contexto de la epidemia de COVID-19:

a) Estudios de caracterización de la enfermedad y su diagnóstico

Respecto a estos estudios surgieron preguntas como "¿Cuál es la tasa de letalidad en cada país de la región?" “¿Cuáles son los marcadores de pronóstico clínico?” “¿Hay seguimiento a la curva de contagio o tenemos una mirada parcial?" Se necesitan estudios de caracterización en diferentes entornos y poblaciones. Los panelistas mencionaron la necesidad de desarrollar capacidades locales para realizar pruebas diagnósticas, validar pruebas rápidas con alta sensibilidad y especificidad para aumentar la capacidad de testeo. Asimismo, destacaron la necesidad de capacitación para ver qué tipos de medios o tipo de transporte se pueden utilizar sin requerimientos de cadena de frío para poder testear en los lugares más rezagados de nuestros países.

b) "Efectos adversos no intencionados de las políticas públicas implementadas"

Se mencionaron las enfermedades crónicas como la tuberculosis y la infección por $\mathrm{VIH}$, otras enfermedades infecciosas como el dengue, pero también temas como la educación y los retos de la educación virtual, la seguridad alimentaria y los efectos que van a surgir posteriormente en países que ya se encuentran en situación precaria. Hubo también particular énfasis en la salud materno infantil. Varios participantes en el taller expresaron su preocupación con respecto a la salud reproductiva y la atención de salud durante el embarazo. Una participante comentó: “¿Cómo está afectando la pandemia el acceso y la utilización de la atención prenatal y la atención al parto? ¿Se está observando un aumento de la transmisión perinatal de VIH y sifilis y de la mortalidad materna y neonatal?" Asimismo, se mencionó como un asunto de interés, el efecto de la pandemia en la violencia doméstica y el aumento que se está observando de embarazos forzados en adolescentes. Dado que el aborto no es legal en muchos de los países de LAC, varios participantes resaltaron la brecha de generar guías de cómo afrontar estos 
Cuadro 1. Clasificación de las prioridades de investigación dentro de la epidemia de COVID-19 en América Latina y el Caribe a corto y a largo plazo dentro de las categorías de la OMS, encuesta en línea, abril - julio de 2020.*

Categorías
mistoria natural del virus, trans-
y diagnóstico
Investigación animal y ambiental
sobre el origen del virus y me-
didas de gestión en la interfaz
humano-animal

Estudios epidemiológicos

Gestión clínica

Prevención y control de infec- 1 ciones, incluida la protección de los trabajadores de la salud

Candidato a Investigación y de- 1 sarrollo terapéutico

Investigación y desarrollo de va- 1 cunas candidatas

2

3

2
No. Prioridad a Corto Plazo (según las establecidas por Prioridad a Largo Plazo (según las establecidas por $\mathrm{OMS}^{2}$ y Academia Africana de Ciencias ${ }^{13}$ ) OMS $^{2}$ y Academia Africana de Ciencias ${ }^{13}$ )

- Apoyar el trabajo de desarrollo de pruebas de antíge- - Apoyar el desarrollo de productos de diagnóstico para nos de campo más baratas, rápidas y fáciles de usar mejorar los procesos clínicos. (para la detección de virus)

2 - Apoyar el desarrollo de productos de diagnóstico para - Apoyar el trabajo para desarrollar pruebas de anticuermejorar los procesos clínicos. pos de campo más baratas, más rápidas y más fáciles de usar (para determinar la exposición).

3 - Apoyar el trabajo para desarrollar pruebas de anticuerpos de campo más baratas, más rápidas y más fáciles de usar (para determinar la exposición). ${ }^{* *}$

- Apoyar el trabajo de desarrollo de pruebas de antígenos de campo más baratas, rápidas y fáciles de usar (para la detección de virus)

- Mejorar la comprensión de los factores de riesgo socioeconómicos y de comportamiento para el desbordamiento y la transmisión entre animales y humanos

- Estudios ambientales del SARS-Cov-2, incluidas las prácticas de gestión de desechos y aguas residuales

- Diseñar y probar estrategias adecuadas de reducción - Diseñar y probar estrategias adecuadas de reducción de de riesgos en la interfaz hombre-animal-ambiente. riesgos en la interfaz hombre-animal-ambiente.

1 - Describir las dinámicas de transmisión de COVID-19 y comprender la propagación de la enfermedad a nivel nacional, regional y mundial. **

2 - Evaluar el impacto de las medidas de control y mitigación, por ejemplo, la elaboración de modelos para estimar los efectos de las medidas de distanciamiento social y otras intervenciones no farmacéuticas. ${ }^{*}$

- Evaluar el impacto de las medidas de control y mitigación, por ejemplo, la elaboración de modelos para estimar los efectos de las medidas de distanciamiento social y otras intervenciones no farmacéuticas. ${ }^{* *}$

3 - Describir la gravedad de la enfermedad y la susceptibilidad para facilitar una respuesta clínica y de salud pública eficaz a COVID-19 - identificar los grupos de alto riesgo de infección grave

1 - Determinar las intervenciones que mejoren el resultado clínico de los pacientes con COVID-19

- Definir la historia natural de la infección por SARSCoV-2 en COVID-19 a través de una cuidadosa y completa descripción clínica y de laboratorio de los casos.

2 - Determinar las estrategias óptimas de práctica clínica para mejorar los procesos de atención (por ejemplo, elaborar criterios para el diagnóstico temprano, cuándo dar de alta, cuándo utilizar terapias adyuvantes para los pacientes y los contactos).**

- Definir la historia natural de la infección por SARS- - Determinar las intervenciones que mejoren el resultado CoV-2 en COVID-19 a través de una cuidadosa y completa descripción clínica y de laboratorio de los casos.

- Optimizar la eficacia del EPP y su uso para reducir el - Comprender la eficacia de las estrategias de control del riesgo de transmisión en los entornos de atención de la salud y de la comunidad. movimiento para prevenir la transmisión secundaria en los entornos de atención de la salud y de la comunidad

- Comprender la eficacia de las estrategias de control del movimiento para prevenir la transmisión secundaria en los entornos de atención de la salud y de la comunidad ${ }^{* *}$

- Investigación para apoyar el fortalecimiento de los sistemas de salud y el fomento de la capacidad de recuperación después del brote ${ }^{\star *}$

- Comprender los factores de comportamiento y culturales que influyen en el cumplimiento de las medidas de EPI basadas en la evidencia.

- Desarrollar mecanismos de apoyo a la colaboración - Apoyar la ciencia básica para identificar nuevos objeticoordinada para llevar a cabo ensayos clínicos para vos de medicamentos evaluar de la seguridad/eficacia de las terapias.*

- Apoyar la ciencia básica para identificar nuevos objeti- - Desarrollar un Protocolo Maestro Multicéntrico para evavos de medicamentos ${ }^{* *}$

luar la eficacia y la seguridad.

3 - Investigaciones sobre la potencia del antisuero convaleciente como opción terapéutica

- Desarrollar mecanismos de apoyo a la colaboración coordinada para llevar a cabo ensayos clínicos para evaluar de la seguridad/eficacia de las terapias.

- Desarrollar un Protocolo Maestro Multicéntrico para evaluar la eficacia y la seguridad.

- Elaborar un protocolo maestro multinacional para la evaluación de las vacunas de la fase $2 \mathrm{~b} / \mathrm{fase} 3 \mathrm{a}$ fin de determinar si las vacunas candidatas son seguras y eficaces antes de su distribución generalizada.

2 - Desarrollo de la capacidad para la ciencia básica y el desarrollo preclínico de nuevas vacunas

- Identificar la correlación y la protección del Programa Sin dato.

Ampliado de Inmunización y otras vacunas, por ejemplo, la vacuna BCG

3

- Identificación de candidatos para la evaluación clínica - Identificar la correlación y la protección del Programa además de los ya priorizados. la vacuna $B C G$. 
Consideraciones éticas para la 1 investigación
- Identificar las principales lagunas de conocimiento y las prioridades de investigación en relación con las cuestiones éticas derivadas de las medidas restrictivas de salud pública propuestas (por ejemplo, cuarentena, aislamiento, cordón sanitario).

2 - Definir un marco de gobernanza de la investigación que permita una colaboración eficaz y ética entre múltiples interesados, entre ellos la OMS, la comunidad mundial de investigadores, los expertos en la materia, los funcionarios de salud pública, los financiadores y los especialistas en ética.

3 - Establecer procesos para acelerar el examen ético de las propuestas de investigación relacionadas con COVID-19** puesta al brote
- Promover la priorización de las necesidades de conocimiento según la dinámica de la epidemia.

\section{- Examinar las formas óptimas de comunicación sobre posibles intervenciones en entornos urbanos de alta densidad y baja condición socioeconómica \\ - Asegurar que los conocimientos se produzcan de acuer- do con las necesidades locales, nacionales y regionales.} posibles intervenciones en entornos urbanos de alta densidad y baja condición socioeconómica*

3 - Investigar las formas de asegurar la transparencia del flujo de información y mitigar la información falsa difun- miento según la dinámica de la epidemia.

\footnotetext{
*Se presentan las 3 primeras prioridades, ordenadas de mayor (1) a menor (3) prioridad.

**Prioridades reiteradas también en el taller.

*Prioridades de investigación mayormente destacadas.
}

casos y la necesidad de aumentar la provisión de anticonceptivos orales en los centros de atención.

Un participante del Instituto Dominicano para el Estudio de la Salud Integral y la Psicología Aplicada subrayó la necesidad de seguir investigando en materia de salud mental: "Estamos preocupados por aspectos tales como: 1) Incremento de síntomas psiquiátricos a casusa del incremento de la ansiedad; 2) Impacto del miedo al deterioro de las actividades de la vida diaria, [...] consumo de alcohol, [...] el impacto del distanciamiento social [...] donde la proximidad física es parte de la cultura y tiene códigos socio antropológicos que contradicen las medidas salubristas de distanciamiento social". A su vez, otra participante resaltó la necesidad de tomar "alguna medida para proteger la salud mental del personal de salud". En el contexto de la COVID-19, trabajadores de salud se encuentran con incertidumbre y preocupados por la falta los equipos de protección y las diferentes disonancias de cómo usarlos.

c) Formas óptimas de comunicación

Los panelistas señalaron también la necesidad de evaluar el impacto positivo y negativo (fake news) de los medios de comunicación, que es lo que se comunican a la población y con qué fin. Se resaltó que estudios antropológicos pueden ser útiles para fortalecer la eficacia de las comunicaciones y el impacto en la adherencia hacia las medidas de intervención propuesta como hacia la autopercepción de riesgo y autocuidado.

d) Vigilancia epidemiológica

Los participantes recalcaron que el aumento de los recursos para la vigilancia de COVID19 disminuyó la vigilancia de otras enfermedades. Ante la pregunta: ¿Cuál es la capacidad del sistema de salud para poder responder a la pandemia?, se realizó especial mención a la necesidad de estudios de modelaje para evaluar proyecciones de la epidemia y obtener el umbral de movilización para garantizar una epidemia de niveles "controlables", sobre todo en el contexto que caracteriza a LAC.

e) Servicios y sistemas de salud

Surgieron preguntas como ¿Cuál es el impacto de las intervenciones generadas para COVID en el acceso a los servicios de salud? ¿Qué modelos hay construidos para realizar abordajes intersectoriales y lograr mayor efectividad en la implementación de las medidas de salud publicas implementadas para el COVID? Por lo tanto, el grupo convino unánimemente en que las numerosas incógnitas que rodeaban el tema requerían una investigación urgente. Asimismo, hace falta estudiar la adhesión a las medidas implementadas por los gobiernos y evaluar cómo fortalecer y reestructurar la capacidad del servicio de salud para permitir, por un lado, la atención desde la casa, y por el otro que las personas lleguen al establecimiento de salud solo cuando sea requerido.

\section{f) Estudios antropológicos}

Para ello, primero hay necesidad de entender ¿Cuál es la percepción del riesgo de COVID19? Esto es clave a medida que se dé el retiro paulatino del confinamiento para tener estrategias adecuadas a esta percepción. Para que estos abordajes sean efectivos y pertinentes, se hizo especial mención a la necesidad del aporte comunitario y participación social.

g) Necesidad de cooperación e interdisciplinariedad

"¿Cómo lograr una integración entre organismos a nivel local y regional?" Una participante de Argentina recalcó que "el rol de las ONG en esta pandemia podría ser de gran importancia por su llegada a la sociedad civil en este abordaje de aporte comunitario" Esto desató un debate. Los panelistas acordaron que las ONG tienen un rol fundamental en ayudar a lograr una coordinación multisectorial e interdisciplinaria. Asimismo, se mencionó la necesidad de un enfoque interdisciplinario con equipos de trabajo que incluya psicólogos, médicos, gerentes administrativos de los servicios, economistas, seguridad y justicia, e incluso meteorólogos. A nivel internacional hace falta más integración regional, especialmente para cuando los países comiencen a levantar las restricciones fronterizas. Se resaltó la necesidad de investigar con base a experiencias pasadas para desarrollar protocolos de manejo intrafronterizo.

El Cuadro 2 muestra cómo se agruparon las prioridades identificadas en este estudio en relación a las 9 categorías de la agenda de la OMS².

Se observa que las prioridades de investigación destacadas por los ponentes y participantes del taller, se alinean en gran parte con aquellas señaladas por el cuerpo de especia- 
listas convocados por la OMS. Asimismo, se identificaron prioridades existentes que requieren ahora un mayor énfasis en la investigación como ser la necesidad de evaluar los efectos adversos no intencionados de las intervenciones de salud pública en la salud materna e infantil, así como también en la salud mental. También se identificaron nuevas prioridades que no figuran en la hoja de ruta de la OMS ni surgieron en la lista de la AAS, como ser la falta de recursos humanos en el sector de la investigación (Cuadro 2).

\section{DISCUSIÓN}

En un contexto global que discute el tema de la sostenibilidad, las Naciones Unidas firmaron en 2015 la Agenda 2030. ${ }^{16}$ El Objetivo de Desarrollo Sostenible 3, "Asegurar una vida saludable y promover el bienestar de todos y todas, a todas las edades", tiene metas relevantes cuando se trata de emergencia de pandemias. Siguiendo estos lineamientos, la Organización Panamericana de la Salud (OPS) aprobó una agenda específica en 2018. ${ }^{17}$ Ambos documentos destacan la promoción del acceso a servicios e insumos de salud, el apoyo a la investiga- ción y desarrollo de vacunas y medicamentos, el aumento del financiamiento en salud y el fortalecimiento de la capacidad de los países en el desarrollo de la vigilancia en salud.

Las necesidades de investigación destacadas en este análisis se incorporan con muchas de las establecidas en los documentos da las Naciones Unidas, de la OPS y de la OMS, con el refuerzo de los sistemas de salud en general, pero específicamente en el desarrollo de insumos de salud que sean accesibles a todos, la capacitación de recursos humanos y el fortalecimiento de las capacidades de vigilancia en salud. Asimismo, la cooperación regional entre los países de LAC se mostró como una vía prometedora para lograr abordar dichas prioridades. Estos elementos refuerzan la tesis de que, para responder a los desafíos globales de salud, es necesario contar con sistemas de salud fortalecidos y con capacidad de realizar trabajo intersectorial.

En 1990, la Comisión en Investigación en Salud para el Desarrollo (Commission on Health Research for Development) declaró que el fortalecimiento de la capacidad de investigación en los países de ingresos bajos y medianos es "uno de los medios más poderosos, eficaces en función de los costos y sostenibles

Cuadro 2. Principales prioridades de investigación dentro de la epidemia de COVID-19 a partir del examen del taller virtual en relación con lo establecido por la OMS, abril - julio de 2020.

\begin{tabular}{|c|c|c|}
\hline \multicolumn{2}{|r|}{ Categorías establecidas por $\mathrm{OMS}^{2}$} & \multirow{2}{*}{$\begin{array}{l}\text { Prioridades mencionadas en el taller categorizadas } \\
\text { - Inmunidad y su duración } \\
\text { - Capacidades locales para realizar pruebas diagnósticas } \\
\text { - Pruebas seguras y eficaces }\end{array}$} \\
\hline $1-$ & Historia natural del virus, transmisión y diagnóstico & \\
\hline $2-$ & $\begin{array}{l}\text { Investigación animal y medioambiental sobre el origen del virus, y } \\
\text { medidas de gestión en la interfaz humano-animal }\end{array}$ & - No aplica \\
\hline $3-$ & Estudios epidemiológicos & $\begin{array}{l}\text { - Modelos y proyección de la pandemia } \\
\text { - Estudios observacionales } \\
\text { - Estudios sobre transmisión en asintomáticos } \\
\text { - Investigación sobre las políticas públicas de salud actualmente puestas a } \\
\text { cabo* }\end{array}$ \\
\hline 4- & Manejo clínico & - Calidad de la atención \\
\hline $5-$ & Prevención y control de infecciones & - Vigilancia epidemiológica* \\
\hline 6- & Candidato terapéutico I+D & - Evaluación de medidas farmacológicas \\
\hline 7- & Vacunas candidatas I+D & - No aplica \\
\hline 8- & Consideraciones éticas para la investigación & $\begin{array}{l}\text { - La importancia de los comités éticos para acompañar y asesorar técnica- } \\
\text { mente los estudios de investigación }\end{array}$ \\
\hline 9- & Las ciencias sociales en la respuesta al brote & $\begin{array}{l}\text { - Falta de estudios antropológicos }{ }^{\star} \\
\text { - Percepción de riesgo } \\
\text { - Comunicación* } \\
\text { - Participación comunidad } \\
\text { - Necesidad de cooperación e interdisciplinariedad* } \\
\text { - Sistemas y servicios de salud incluyendo } \\
\text { + recursos humanos } \\
\text { + Investigación sobre las políticas públicas de salud actualmente } \\
\text { puestas a cabo* } \\
\text { - Los efectos adversos no intencionados de las intervenciones de salud } \\
\text { pública en: } \\
\text { + otras enfermedades infecciosas (e.g. Dengue) } \\
\text { + en las enfermedades crónicas } \\
\text { + en la salud materna, reproductiva e infantil }{ }^{\star} \\
\text { + en la salud mental* }\end{array}$ \\
\hline & vas Prioridades & $\begin{array}{l}\text { - Carencia de herramientas de investigación en español } \\
\text { - Falta de recursos humanos (cantidad y capacidad) en el sector de la investi- } \\
\text { gación* }\end{array}$ \\
\hline
\end{tabular}


para promover la salud y el desarrollo". ${ }^{18}$ Hasta la fecha, a pesar de los esfuerzos internacionales, los estudios de investigación que abordan los problemas de salud locales siguen siendo, en gran parte, dirigidos por países de altos ingresos. ${ }^{19} \mathrm{~L}$ a pandemia subraya con demasiada claridad brechas en el financiamiento de investigación en nuestra región y falta de disponibilidad de instituciones de salud global en adaptar sus herramientas a distintos contextos, ya que cuestiones como vulnerabilidades, inequidades y desarrollo tiene distintos matices en el Sur Global. Estas brechas impactan en investigaciones observacionales, descripción de las enfermedades y, posteriormente, establecer ensayos clínicos normalizados de carácter reglamentario para evaluar posibles medicamentos y vacunas.

La necesidad de adoptar medidas para el desarrollo de la capacidad de investigación en materia de salud en LAC ha sido abordada a nivel regional por la OPS desde 2009 cuando publicó por primera vez un documento de política de investigación. ${ }^{20} \mathrm{Si}$ bien todos los elementos básicos de un sistema de investigación son necesarios y están interrelacionados, podría decirse que la clave para desarrollar una capacidad local sostenible proviene de contar con equipos bien capacitados y con personas que puedan demostrar su liderazgo en la investigación, cualquiera que sea su función. ${ }^{21} \mathrm{El}$ aumento de la inversión en ciencia y tecnología en LAC debería ser un objetivo para la acción política nacional e internacional, hoy más que nunca.

El tema de la capacitación de los recursos humanos se muestra como una oportunidad para la circulación de conocimientos y prácticas, además de metodologías entre distintos actores, países, contextos epidémicos y sociales. De esa forma, se ampliaría el acceso a conocimientos y a las respuestas concretas a los desafíos puestos por la pandemia en LAC en su diálogo global y sus mejores evidencias. En respuesta, La Red de Salud Global busca ser una herramienta facilitadora para fortalecer las capacidades de investigación y promover la cooperación.

Para ello, en el mes de mayo de 2020 se realizó una invitación abierta para que todos los involucrados en investigación se pongan en contacto ofreciendo una plataforma para la creación de redes, el aprendizaje compartido y la implementación de estudios de investigación. Así, se establecieron los "Grupos de trabajo sobre COVID-19", una iniciativa innovadora internacional que aborda el debate y la continua creación de consenso y acción en torno a las prioridades de investigación identificadas. Utilizando un sistema de desarrollo de crowdsourcing y una herramienta diseñada específicamente, los miembros comparten sus enfoques, procedimientos operativos estándar y recursos de capacitación para apoyar una implementación más rápida de los estudios de investigación durante esta pandemia. Este método innovador invita y asegura la aportación multidisciplinar para maximizar el rigor del diseño y una mayor aplicabilidad. Estos protocolos se publicarán para que todos los utilicen.

Instituciones y centros como La Red de Salud Global, sirven como plataformas interdisciplinarias e intersectoriales, facilitadores del ecosistema que catalizan la investigación, ${ }^{22}$ haciendo posible el acceso a servicios de salud más inclusivos, efectivos y asequibles. ${ }^{12}$ Esperamos que los hallazgos expuestos en esta investigación ayuden a generar más iniciativas inno- vadoras interdisciplinarias para determinar e impulsar métodos más rápidos, elevar los estándares y hacer que la investigación sea más eficiente y accesible para luego llevar esos hallazgos a la práctica.

En relación a las dos únicas prioridades identificadas por los expertos de OMS que no figuran en el análisis del taller interactivo (Investigación animal y medioambiental sobre el origen del virus y medidas de gestión en la interfaz humano-animal y vacunas candidatas $I+D$ ), una de las posibles razones para esto es que, en el momento del taller, diversos países de LAC ya estaban involucrados en ensayos clínicos y proyectos para el desarrollo de vacunas. Otra posible explicación, es el perfil de los ponentes - y por consecuencia de la audiencia - formada por epidemiólogos y especialistas en salud materno-infantil desde perspectivas de integralidad y equidad. De esa manera, estos dos temas se alejan de sus campos usuales de investigación y quizás por eso no se destacaron como prioridades en sus presentaciones.

Entre las limitaciones de la encuesta, se encuentra el hecho de que en las preguntas se mantuvo el orden original de prioridades dentro de cada uno de las 9 categorías, lo que puede haber influido en la clasificación dada por los encuestados. Sin embargo, dado que se utilizó una metodología mixta, que el taller fue abierto, y que los investigadores participantes (de diferentes contextos de salud) pudieron hacer comentarios con respecto a las prioridades de investigación actuales, más allá del alcance de la Guía de Investigación de la OMS, consideramos que estos datos son relevantes.

A pesar de las limitaciones, en conjunto, sugerimos que los resultados y el análisis aquí presentado, son relevantes para poner de relieve los aspectos en los que los financiadores e investigadores deberían priorizar, así como la identificación de posibles nuevas áreas que deberían abordarse. Esperamos que los hallazgos expuestos en esta investigación ayuden a orientar la toma de decisiones y a generar más iniciativas como las de la Red, específicas para LAC, a través del amplio sistema de investigación, incluyendo a los socios multilaterales, los financiadores de la investigación, los profesionales de la salud pública, los clínicos y la sociedad civil.

En conclusión, para nuestra región, la pandemia se presenta como una oportunidad para la cooperación regional e internacional en diversos niveles y se hace necesario que establezcamos redes de conocimiento para responder a estos desafíos comunes y promover el desarrollo y equidad en ciencia y tecnología para lograr mejores y más robustas respuestas a las amenazas sanitarias. Pensamos que este es un objetivo estratégico para la salud global en el marco de la pandemia de COVID-19.

\section{CONTRIBUCIONES}

NFC, FB, BB diseñaron el estudio, la estrategia del taller virtual "COVID-19 para países de habla hispana" y el análisis de los resultados, tanto del taller como de la encuesta en línea. Todos los autores contribuyeron al desarrollo del taller y participaron en el mismo. NFC, FB, JA, BB, lideraron la redacción del artículo. Todos los autores contribuyeron y aprobaron la versión final del artículo. 


\section{AGRADECIMIENTO}

Reconocemos y agradecemos a los participantes en el taller interactivo y en la encuesta en línea por sus valiosas contribuciones. Se agradece al Dr. Carlos J. Fajardo, Centro de Cáncer Emma Romero de Callejas, Tegucigalpa, Honduras, por la revisión crítica del manuscrito. La Red cuenta con financiamiento de la Fundación Bill y Melinda Gates (https://www. gatesfoundation.org/ Número de la subvención: OPP1169808). El COVID-19 HUB específicamente se financia con una donación de UK Research and Innovation (https://www.ukri.org/ Número de la donación: MC_PC_19073). Fiocruz es financiado por Wellcome Trust, Proyecto número 218750/Z/19/Z.

\section{REFERENCIAS}

1. World Health Organization. WHO Director-General's opening remarks at the media briefing on COVID-19 - 11 March 2020 [Internet]. Geneva: WHO; 2020. [citado 11 junio 2020]. Disponible en: https://www.who.int/dg/speeches/detail/who-director-general-s-opening-remarks-at-the-media-briefingon-covid-19-- 11-march-2020.

2. World Health Organization. A Coordinated Global Research Roadmap: 2019 Novel Coronavirus [Internet]. Geneva: WHO; 2020.[citado 20 julio 2020]. Disponible en https://www.who.int//lueprint/priority-diseases/key-action/Coronavirus_Roadmap_V9.pdf?ua=1

3. Garland Mahler A. Global South. Oxford Bibliographies in Literary and Critical Theory, ed. Eugene O'Brien. New York: Oxford University Press; 2017. DOI: 10.1093/obo/9780190221911-0055

4. Ventura DFL, Ribeiro H, Giulo GM, Jaime PC, Nunes J, Bógus CM, et al. Desafíos da pandemia de COVID-19: por uma agenda brasileira de pesquisa em saúde global e sustentabilidade. Cad Saúde Pública. 2020; 36(4):e00040620.

5. World Health Organization. Coronavirus disease (COVID-19) pandemic, 2020 May 4. [Internet]. Geneva: WHO; 2020. [citado 05 mayo 2020]. Disponible en: https://www.who.int/emergencies/diseases/novel-coronavirus2019External Link

6. Lang T. The Global Health Network and the resources available for capacity building [Internet]. 2017. [citado 11 junio 2020]. Disponible en: https://www. glopid-r.org/ressources/the-global-health-network-and-the-resources-available-for-capacity-building/

7. Alger J, Furtado T, van Loggerenberg F. The Global Health Network: Plataforma virtual para capacitación en ética de la investigación. Rev Med Hondur. 2015; 83 (3-4): 165-166.

8. Furtado T, Franzen S, van Loggerenberg F, carn G, Grahek S, McBride M, et al. Strengthening neglected tropical disease research through enhancing research-site capacity: an evaluation of a novel web application to facilitate research collaborations. PLoS Negl Trop Dis. 2014;8(11):e3225.

9. Franzen SR, Chandler C, Lang T. Health Research Capacity Development in Low- and Middle-Income Countries: Reality or Rhetoric? A Systematic Meta-Narrative Review of the Qualitative Literature. BMJ Open. 2017; 7(1): e012332

10. Hajduk GK, Jamieson NE, Baker BL, Olesen OF, Lang T. It is not enough that we require data to be shared; we have to make sharing easy, feasible and accessible too! BMJ Glob Health. 2019;4(4):e001550.

11. Horby PW, Endzt H, Muyembe-Tamfum JJ, van Griensven J, Gevao S, Goossens $\mathrm{H}$, et al. Ebola: Europe-Africa research collaborations. Lancet Infect Dis [Internet]. 2015[citado 11 junio 2020];15(11):1258-1259. doi:10.1016/S14733099(15)00375-8. Erratum in: Lancet Infect Dis. 2015;15(12):1375

12. van Niekerk $L$. Social innovation in health In: van Niekerk $L$, Chater $R, N a-$ ydenova E, Lim J, Chamas L, Manderson L, Gilson L, Peeling R, Hartigan $\mathrm{P}$, Bonnici F, editors. Social innovation in health: case studies and lessons learned from low- and middle-income countries. Geneva: World Health Organization; 2017. P.10-12.

13. The African Academy of Sciences. Research and Development goals for COVID-19 in Africa: The African Academy of Sciences Priority Setting Exercise [Internet]. Nairobi: AAS; 2020. [citado 28 junio 2020]. Disponible en: https://www.aasciences.africa/sites/default/files/2020-04/Research\%20 and\%20Development\%20Goals\%20for\%20COVID-19\%20in\%20Africa.pdf

14. Tibenderana J, Alia J, Hamade P, Walker R, Feune De Colombi N, Al-Rawni Z, et al. Malaria and COVID-19: A rapid determination of unknowns and call for research. Paper finalised. MedRxiv Pre-print server on 10th July 2020.

15. Norton A, De La Horra Gozalo A, Feune de Colombi N, et al. The remaining unknowns: a mixed methods study of the current and global health research priorities for COVID-19. BMJ Global Health. 2020;5:e003306.

16. United Nations. Transforming our world: The 2030 agenda for sustainable development. [Internet]. N.Y.: UN; 2019. [citado 07 julio 2020]. Disponible en: https://sdgs.un.org/2030agenda

17. Organización Panamericana de la Salud . Organización Mundial de la Salud. Agenda de Salud Sostenible para las Américas 2018-2030 [Internet]. Washington D.C.: OPS/OMS; 2019. [citado 07 julio 2020]. Disponible en: https:// www.paho.org/es/agenda-salud-sostenible-para-americas-2018-2030

18. Commission on Health Research for Development. Health Research - Essential Link to Equity in Development. Asia-Pacific J Public Heal. 1990;4(4):1990.

19. Jones N, Bailey M, Lyytikäinen M. Research capacity strengthening in Africa Trends, gaps and opportunities. London; 2007.

20. Julé A, Furtado T, Boggs L, van Loggerenberg F, Ewing V, Vahedi M, et al. Developing a globally applicable evidence-informed competency framework to support capacity strengthening in clinical research [published correction appears in BMJ Glob Health. 2017;7:2(3):e000229corr1]. BMJ Glob Health. 2017;2(2):e000229.

21. Buss PM, Tobar S. COVID-19 and opportunities for international cooperation in health. Cadernos de Saúde Pública, Rio de Janeiro, 2020: 36(4): 1-3.

22. Niekerk L, Mathanga P, Juban N, Castro-Arroyave DM, Balabanova D. Universities as catalysts of social innovation in health systems in low-and middleincome countries: a multi-country case study. Infect Dis Poverty. 2020; 9:90. https://doi.org/10.1186/s40249-020-00684-5

ABSTRACT. Background: The Global Health Network (www. tghn.org) established in January 2020 a community of practice to address research on COVID-19 in low/middle-income countries. Objective: To identify the COVID-19 research gaps that require urgent attention in Latin America and the Caribbean (LAC) and to establish a local open community of practice to support its implementation. Methods: Mixed methods exploratory study. LAC-specific results, from an online Survey (quantitative approach) that assessed whether the WHO's priority research agenda for COVID-19 is still relevant, were analysed by asking participants to rank their top three options for short- and long-term priorities. An open virtual workshop (qualitative approach) was also organised and recorded. A pragmatic thematic analysis was conducted based on the panellists' presentations and audience questions and comments. A coding framework was generated using inductive and then deductive approaches following the WHO agenda. Results: There were 223 participants from 22 countries. A consensus was identified on priority research and innovation issues for LAC, within and outside the WHO agenda, being a high priority the necessity of social sciences studies to support biomedical scientists. Discussion: As COVID-19 cases continue to increase in LAC, we believe that our findings are useful in guiding both research networks in planning studies and funders in their decisions to allocate resources to research and innovation.

KEYWORDS. Caribbean region, Coronavirus infection, Health priorities, Latin America, Professional practice gaps, Research. 